\title{
A three-dimensional computational fluid dynamics model of shear stress distribution during neotissue growth in a perfusion bioreactor ${ }^{\dagger}$
}

$$
\text { Guyot } \mathrm{Y}^{1,2} \text {, Luyten } \mathrm{FP}^{1,3} \text {, Schrooten } \mathrm{J}^{1,4} \text {,Papantoniou } \mathrm{I}^{1,3^{*}} \text {, Geris } \mathrm{L}^{1,2,5^{*}} \text {. }
$$

${ }^{1}$ Prometheus, Division of Skeletal Tissue Engineering, KU Leuven, Onderwijs en Navorsing 1 (+8), Herestraat 49 - PB813, B-3000, Leuven, Belgium.

${ }^{2}$ Biomechanics Research Unit, Universite de Liège, Chemin des Chevreuils 1 - BAT 52/3, B4000 Liège, Belgium

${ }^{3}$ Skeletal Biology and Engineering Research Center, KU Leuven, Onderwijs en Navorsing 1 (+8), Herestraat 49 - PB813, B-3000 Leuven, Belgium.

${ }^{4}$ Department of Materials Engineering, KU Leuven, Kasteelpark Arenberg 44 - PB 2450, B3001 Leuven, Belgium

${ }^{5}$ Biomechanics Section, KU Leuven, Celestijnenlaan 300C - PB 2419, B-3001 Leuven, Belgium

Address for Correspondence:

Yann Guyot; Email: yguyot@ulg.ac.be

*these authors share last authorship.

${ }^{\dagger}$ This article has been accepted for publication and undergone full peer review but has not been through the copyediting, typesetting, pagination and proofreading process, which may lead to differences between this version and the Version of Record. Please cite this article as doi: [10.1002/bit.25672]

This article is protected by copyright. All rights reserved Received November 23, 2014; Revision Received March 18, 2015; Accepted June 05, 2015 


\begin{abstract}
Bone tissue engineering strategies use flow through perfusion bioreactors to apply mechanical stimuli to cells seeded on porous scaffolds. Cells grow on the scaffold surface but also by bridging the scaffold pores leading a fully filled scaffold following the scaffold's geometric characteristics. Current computational fluid dynamic approaches for tissue engineering bioreactor systems have been mostly carried out for empty scaffolds. The effect of 3D cell growth and extracellular matrix formation (termed in this study as neotissue growth), on its surrounding fluid flow field is a challenge yet to be tackled. In this work a combined approach was followed linking curvature driven cell growth to fluid dynamics modeling. The level-set method (LSM) was employed to capture neotissue growth driven by curvature, while the Stokes and Darcy equations, combined in the Brinkman equation, provided information regarding the distribution of the shear stress profile at the neotissue/medium interface and within the neotissue itself during growth. The neotissue was assumed to be micro-porous allowing flow through its structure while at the same time allowing the simulation of complete scaffold filling without numerical convergence issues. The results show a significant difference in the amplitude of shear stress for cells located within the micro-porous neo-tissue or at the neotissue/medium interface, demonstrating the importance of taking along the neotissue in the calculation of the mechanical stimulation of cells during culture. The presented computational framework is used on different scaffold pore geometries demonstrating its potential to be used a design as tool for scaffold architecture taking into account the growing neotissue. This article is protected by copyright. All rights reserved
\end{abstract}

Keywords: bioreactor, scaffold, computational fluid dynamics, tissue growth, level-set method 


\section{Introduction}

Perfusion bioreactors have been extensively explored for the production of bone forming tissue engineered constructs by supporting cell growth, metabolism, and differentiation. Attempts to culture in vitro three dimensional (3D) tissue engineered (TE) constructs in static conditions typically resulted in an outer shell of viable cells, while the inner core became necrotic due to limited diffusive delivery of nutrients (Griffith et al., 2005). Using bioreactors, cell proliferation and cell survival have been enhanced substantially when compared with static setups, by improving mass transport of oxygen and nutrients throughout the TE construct during cell growth (Martin, Wendt et al. 2004). However, fluid flow exerts stresses on the cultured cells affecting functions such as migration, proliferation and apoptosis (McCoy and O'Brien 2010). Furthermore shear stress can determine lineage commitment (Song, Dean et al. 2013) but also promote osteogenic differentiation of bone marrow stromal, periosteum and adipose derived mesenchymal stem (stromal) cells, and enhance extra-cellular matrix deposition (McCoy and O'Brien 2010; Rauh, Milan et al. 2011; Papantoniou, Chai et al. 2013).

In order to understand and characterize complex flow fields which are developed throughout regular and irregular scaffolds during cell culture in bioreactors, computational fluid dynamics (CFD) modeling has been extensively used in tissue engineering (for review see (Hutmacher and Singh 2008)). The quantification of flow-associated shear stresses as well as their spatial distribution within various 3D scaffold geometries has been thoroughly investigated in perfused bioreactor setups (Raimondi, Boschetti et al. 2004; Porter, Zauel et al. 2005; Boschetti, Raimondi et al. 2006; Cioffi, Boschetti et al. 2006; Jungreuthmayer, Donahue et al. 2009; Maes, Ransbeeck et al. 2009; Voronov, VanGordon et al. 2010). In previous studies, local shear stresses were defined as a function of flow rate of the culture medium, bioreactor configuration, porosity and porous scaffold micro-architecture (Voronov, 
VanGordon et al. 2010; Pham, Voronov et al. 2012). However the majority CFD studies to date used empty scaffold geometries to calculate shear stress magnitude and distribution across the empty scaffold surface i.e. wall shear stress. They did not take into account the dynamics of the changing 3D environment as a result of cell/tissue growth. Efforts to mimic 3D cell growth on regular scaffolds were limited to a swelling of the scaffold struts (Lesman, Blinder et al. 2010) or the use of a growing biomass (Nava, Raimondi et al. 2013), in both cases modeling the cell layer to be impermeable whilst not being able to reach a full filling of the scaffold void.

$3 \mathrm{D}$ cell growth and neotissue formation starts with $2 \mathrm{D}$ cell migration and proliferation on the strut surface, followed by cells bridging the scaffold void spaces and growing in the third dimension with matrix deposition. This will eventually lead to complete scaffold void filling as evidenced recently via synchrotron and nano-CT imaging (Voronov, VanGordon et al. 2013; Papantoniou, Sonnaert et al. 2014). This means that estimation of wall shear stress values in empty scaffold geometries would only be indicative of the shear stress experienced by cells during early culture time points. A current bottleneck to further advance and utilize CFD strategies in the TE field is the inclusion of a domain composed of cells and extracellular matrix (a growing permeable neotissue) on real scaffold geometries as recently stated (Hendrikson, van Blitterswijk et al. 2014). This way the whole range of fluid-flow associated stresses exerted on cells during neotissue growth could be determined.

In this paper we present a computational strategy that will help understand shear stress magnitude and distribution along the fluid-neotissue interface but also within the permeable 3D neotissue during growth in a perfusion bioreactor. To achieve this, the level-set method (LSM) was employed capturing neotissue growth on 3D macro-porous Titanium scaffolds over time leading to complete scaffold pore closure (filling). Computational neotissue growth was driven by the local curvature as previously described (Bidan, Wang et al. 2013; Guyot, 
Papantoniou et al. 2014). At various degrees of scaffold filling, the steady Brinkman equation was used to continuously describe both flow through a micro-porous medium (neotissue) and free flow through the macro-porous scaffold void. This shear stress mapping will allow to eventually link experimentally determined biological responses (i.e. cell phenotype) to the actual shear stress regime that cells experience during 3D neotissue growth in perfusion bioreactors.

\section{Materials and Methods}

\subsection{Ti6Al4V scaffolds}

Regular, 3D additive manufactured Ti6Al4V scaffolds (diameter $6 \mathrm{~mm}$, height $6 \mathrm{~mm}$ ) were produced in-house using selective laser melting, based on a diamond-shaped unit cell (Pyka, Burakowski et al. 2012) (Figure 1B) and a square unit cell (Van Bael et al. 2012) (Figure 1C). Strut diameter for both scaffolds was $200 \mu \mathrm{m}$. Prior to use, the scaffolds were ultrasonically cleansed for $10 \mathrm{~min}$ over three wash steps in acetone, ethanol and distilled water. Subsequently they received an alkali treatment with 5M sodium hydroxide (Sigma-Aldrich) at $60^{\circ} \mathrm{C}$ for $24 \mathrm{~h}$, rinsed with distilled water and finally sterilized in a steam autoclave. Prior to

cell seeding, all scaffolds were prewetted by vacuum impregnation in cell culture medium for $2 \mathrm{~h}$ in a humidified incubator.

\subsection{Human periost-derived cells (hPDCs)}

hPDCs were isolated from periosteal biopsies of different donors, as described previously (Eyckmans and Luyten 2006). This procedure was approved by the Ethics Committee for Human Medical Research (KU Leuven) and by the patients' informed consent. hPDCs were expanded in Dulbecco's modified Eagle's medium (DMEM) with high glucose (Invitrogen), 
containing 10\% fetal bovine serum (FBS; Gibco), 1\% sodium pyruvate (Invitrogen) and 1\% antibiotic-antimycotic (100 units/ml penicillin, $100 \mathrm{mg} / \mathrm{ml}$ streptomycin and $0.25 \mathrm{mg} / \mathrm{ml}$ amphotericin B; Invitrogen).

\subsection{Scaffold seeding}

The cells were seeded at 5700 cells/cm 2 and passaged at $80-90 \%$ confluence. Prior to the 3D culture experiments, cells were harvested using Triple Express (Invitrogen) and drop-seeded by a single drop onto the scaffolds at a density of 200000 cells/60 $\mu 1$ drop, as performed in earlier studies (Papantoniou, Chai et al. 2013). 45 min after seeding, $60 \mu$ culture medium was added and 135 min later the medium volume was topped up to $1 \mathrm{ml}$. Seeded scaffolds were incubated overnight under standard culture conditions $\left(37^{\circ} \mathrm{C}, 5 \% \mathrm{CO} 2,95 \%\right.$ relative humidity) before being positioned in the perfusion chamber.

\subsection{Bioreactor culture}

Seeded scaffolds were cultured in the perfusion bioreactors for up to 21 days. The perfusion chamber was $26 \mathrm{~mm}$ long with a diameter of $6 \mathrm{~mm}$, holding a single scaffold. This chamber was connected to an individual medium reservoir (disposable $50 \mathrm{~mL}$ Falcon tubes, BD Biosciences) containing $10 \mathrm{~mL}$ of cell culture medium via a Tygon1 (Cole Parmer, Antwerp, Belgium) tubing and a two-stop tubing (BPT, Cole Parmer) connected to a peristaltic pump (IPC-24, Ismatec, SA). Culture medium was refreshed every 2 days for the entire culture period. All seeded scaffolds were positioned $10 \mathrm{~mm}$ from the inlet of the perfusion chamber so that a steady state flow profile was reached as shown in (Papantoniou, Guyot et al. 2014). 


\subsection{SEM imaging}

The micro-porosity of the neotissue between cells and extracellular matrix was approximated by scanning electron microscopy (SEM) coupled with energy-dispersive X-ray (EDAX) analysis (FEI XL30 FEG) at $10 \mathrm{kV}$. Briefly, the cultures were rinsed twice with PBS, fixed with $2.5 \%$ glutaraldehyde (in PBS) for $1 \mathrm{~h}$, and postfixed in $1 \%$ osmium tetroxide for $2 \mathrm{~h}$ before dehydrated in 50\%,75\%, 95\%, and 100\% ethanol solutions. Finally, the samples were chemically dried with hexamethyldisilazane for $3 \mathrm{~min}$ and goldsputtered before SEM analysis.

\subsection{Live/Dead Assay}

The live/dead viability/cytotoxicity kit (Invitrogen) was employed to evaluate qualitatively cell viability and cell distribution and growth by fluorescence microscopy. Constructs were rinsed with $1 \mathrm{~mL}$ PBS after which they were incubated in the staining solution $(0.5 \mathrm{ml}$ Calcein AM and $2 \mathrm{ml}$ Ethidium Homodimer in $1 \mathrm{~mL}$ PBS) for $20 \mathrm{~min}$ in normal cell culture conditions. The constructs were imaged using a Leica M165 FC microscope.

\subsection{Computational Framework}

Neotissue growth with Level-set method (LMS): The evolution of the neotissue topology occurring during the growth process was numerically treated with the LSM which has been developed to deal with moving interfaces. This technique has been used here for its ability to track the front-line between neotissue and void space in a mesh-free manner. In this study, the simulated moving interface $\Gamma$ represents the border between the neotissue domain $\boldsymbol{\Omega}_{\boldsymbol{n} \boldsymbol{t}}$ and the

void domain $\boldsymbol{\Omega}_{\boldsymbol{v}}$. The principle of LSM is based on a continuous distance function $\boldsymbol{\varphi}$ defined on the whole domain $\boldsymbol{\Omega}$ with the following properties: 


$$
\left\{\begin{array}{cc}
\varphi>0 \text { in the neotissue part } \\
\varphi<0 & \text { in the voidpart } \\
\varphi=0 & \text { at the inter face }
\end{array}\right.
$$

To simulate the evolution of the system, the time dependent advection equation (2) is solved with a given velocity $\boldsymbol{v}$.

$$
\frac{\partial \varphi}{\partial t}+\nu \cdot \nabla \varphi=0
$$

Neotissue growth kinetics are curvature-dependent meaning that neotissue grows faster where the curvature is higher and also that it does not grow if the curvature is negative or equal to zero. To tackle this, the advecting velocity $v$ of equation (2) has the following expression:

$$
v=\left\{\begin{array}{c}
-x n \text { if } x>0 \\
0 \text { if } x \leq 0
\end{array}\right.
$$

with $\boldsymbol{n}=\frac{\boldsymbol{\nabla}_{\boldsymbol{\varphi}}}{\left|\boldsymbol{\nabla}_{\boldsymbol{\varphi}}\right|}$ representing the normal at the interface pointing towards the neotissue, and $\boldsymbol{x}=\boldsymbol{\nabla} \cdot \boldsymbol{n}$ representing the mean curvature. Growth simulations were run thanks to the model developed in a previous study (Guyot et al. 2014) providing several neotissue topologies. From these results, geometries corresponding to $10 \%$ to $90 \%$ of scaffold pore filling were taken as starting points for the flow simulations (see below).

Fluid flow model: Neotissue growth leads to changes in morphology affecting the fluid behavior over time. Two fluid flow regimes can be distinguished one within the void but also in the neotissue. In the void part, due to a low Reynolds number, the flow profile can be estimated with Stokes equation (4). 


$$
\left\{\begin{array}{c}
-\mu \Delta u+\nabla p=0 \text { in } \Omega_{v} \\
\nabla \cdot u=0 \text { in } \Omega_{v}
\end{array}\right.
$$

Due to the presence of cells and extracellular matrix in the neotissue part (see Figure 2), the flow profile in this region cannot be estimated with a simple Stokes equation. To address this we consider this region as a porous medium with a given permeability $\boldsymbol{\kappa}_{\mathbf{0}}$ and so the flow profile will be governed by the Darcy equation (5).

$$
\left\{\begin{array}{c}
\frac{\mu}{R_{0}} u+\nabla p=0 \text { in } \Omega_{n t} \\
\nabla \cdot u=0 \text { in } \Omega_{n t}
\end{array}\right.
$$

In (4) and (5), $\mu$ represents the viscosity, $u$ the fluid velocity and $p$ the pressure. Due to the LSM definition of the neotissue topology, the interface would not conform with the fluid computational mesh, and so equations (4) and (5) could be difficult to couple together. One solution to this problem is to model the flow with a penalization technique and the Brinkman equation (6).

$$
\left\{\begin{array}{c}
-\mu \Delta u+\nabla p+\frac{\mu}{\kappa} u=0 \text { in } \Omega \\
\nabla \cdot u=0 \text { in } \Omega
\end{array}\right.
$$

This technique is based on a penalization of the term $K$ in the neotissue domain. For that purpose, a smooth space and time dependent Heaviside function $H$ was calculated with the following equation:

$$
H(\varphi)=\left\{\begin{array}{c}
0, \varphi<-\varepsilon \\
\frac{1}{2}+\frac{\varphi}{2 \varepsilon}+\frac{1}{2 \pi} \sin \left(\frac{\pi \varphi}{\varepsilon}\right),-\varepsilon<\varphi<\varepsilon \\
1, \varphi>-\varepsilon
\end{array}\right.
$$


It should be noted that equation 6 does not take into account time, this corresponds to the fact that the flow is calculated only on given neotissue geometries in order to estimate the mechanical stimuli at that degree of scaffold filling.

The parameter $\boldsymbol{\varepsilon}$ was taken to be equal to $1.5^{*} \mathrm{~h}$, with $\mathrm{h}$ the mesh size. $K$ is then updated according to the following equation:

$$
K(x)=10^{30}(1-H)+K_{0} H
$$

The permeability of the neotissue $\boldsymbol{K}_{\mathbf{0}}$ was calculated using a random fibers approximation to simulate the random unstructured cell and extracellular matrix structure. To tackle this issue, $\boldsymbol{K}_{\mathbf{0}}$ was estimated according to (Nabovati 2009), based on a method to approximate the permeability of a random fibers web, resulting in the following expression for $\boldsymbol{\kappa}_{\mathbf{0}}$ :

$$
K_{0}=0.491 \delta^{2}{\sqrt{\frac{1-\psi_{e}}{1-\psi}-1}}^{2,31}
$$

In this equation, $\boldsymbol{\psi}$ represents the micro-porosity of the neotissue. According to Nabovati et al (2009) $\boldsymbol{\psi}_{e}$ is called the "percolation threshold", and it corresponds to a threshold porosity where fluid flow is permitted through the micro-porous medium. We assumed that such a threshold did not exist, so $\boldsymbol{\psi}_{\boldsymbol{\varepsilon}}$ was set to zero. An important parameter is $\boldsymbol{\delta}$, which corresponds to the average size of the micro-porosity of the neotissue. Two values for $\boldsymbol{\delta}, 25$ and $50 \mu \mathrm{m}$ 
were used based on experimental SEM observations, accounting for the fact that the microporosity could be affected by the culture environment.

Finally, a poiseuille flow corresponding to the average inlet velocity was applied as a dirichlet boundary condition at the entrance of the domain. Symmetry boundary conditions (u.n=0) were applied on each lateral side and a free flow condition at the exit of the domain.

Shear stress estimation: Wall shear stress $\left(\boldsymbol{S S}_{\boldsymbol{s u r f}}\right)$ at the interface between neotissue and void was calculated by:

$$
S S_{s u r f}=\sqrt{\tau_{12}{ }^{2}+\tau_{23}{ }^{2}+\tau_{13}{ }^{2}} \quad \text { where }_{i j}=\mu\left(\frac{\partial u_{i}}{\partial x_{j}}+\frac{\partial u_{j}}{\partial x_{i}}\right)
$$

In addition the shear stress associated with the interstitial flow through the micro-porous neotissue $\left(\boldsymbol{S} \boldsymbol{S}_{\boldsymbol{l n}}\right)$ was calculated based on the method presented in (Whittaker, Booth et al. 2009). The interstitial velocity magnitude $\boldsymbol{u}_{\mathbf{i n}}=|\boldsymbol{u}| / \boldsymbol{\psi}$ was calculated, assuming that the micro-porous neotissue is composed of cylindrical ducts of diameter $\boldsymbol{\delta}$. Hence, the flow profile in each duct can be seen as a Poiseuille flow with a local radial coordinate $r$, and the following velocity profile:

$$
u_{p} \approx 2 u_{i n}\left(1-\left(\frac{2 r}{\delta}\right)^{2}\right)
$$

The inner neotissue shear stress would be:

$$
S S_{I n}=\mu\left|\frac{\partial u_{h}}{\partial r}\right|_{r=\delta / 2} \approx \frac{\theta \mu u_{i n}}{\delta} \approx \frac{\theta \mu|u|}{\delta \phi}
$$


Implementation details: The full implementation of the model presented above has been carried out with Freefem++ (http://freefem.org) (Hecht 2012). For each geometry (diamond and square), the curvature-driven growth model (Guyot et al, 2014) was run on a mesh made of approximatively 1 million tetrahedrons, corresponding to an average element size of 1e-5 $\mathrm{m}$. From these simulations, neotissue topologies corresponding to $10,20, . ., 90 \%$ of scaffold filling were calculated and used as input for the fluid model using the Brinkman equation. The Brinkman equation was solved using the parallelization method of domain decomposition, where the problem is distributed over and solved on 12 cores.

\section{Results \& Discussion}

Live/dead images in Figure 2 show typical 3D cell growth and neotissue formation over time during culture in a perfusion bioreactor for two different scaffold designs with square and diamond pore shapes. In both cases the initial scaffold void was substituted gradually by neotissue. Cells seeded on 3D scaffolds have been seen to be able to bridge across pores of 70 $\mu \mathrm{m}$ entering a 3D growth phase followed by neotissue formation in static conditions (Joly, Duda et al. 2013). In previous studies we have also observed this behavior for bioreactor based cell cultures in scaffolds of larger pores $(\sim 750 \mu \mathrm{m})$ (Papantoniou, Guyot et al. 2014; Papantoniou, Sonnaert et al. 2014). The growing neotissue is a permeable micro-porous structure as can be observed in the SEM image (Figure2). Neotissue growth will subsequently influence the fluid flow profile in the remaining scaffold void space but also the fluid flow derived shear stress distribution and magnitude within the neotissue itself. The flow rate used in this study was $1 \mathrm{ml} / \mathrm{min}$, for which the cells used in this study have been observed to be able to grow up to $100 \%$ scaffold filling in a similar bioreactor set-up (Sonnaert, Papantoniou et al. 2014). In this study, $100 \%$ scaffold filling was indeed reached for both pore shapes as 
shown in Figure2. Their growth under a constant flow rate meant that cells experienced a cascade of increasing shear stresses until complete filling was reached. Two fluid flow regimes could be distinguished, one for free fluid flow and one within the micro-porous neotissue itself. Hence a dynamic multiphase approach was best suited to capture the aforementioned events.

Starting from two different scaffold designs, cell growth was simulated following a curvature dependent growth mechanism (Bidan, Kommareddy et al. 2012; Bidan, Wang et al. 2013; Guyot, Papantoniou et al. 2014). Once coupled with fluid flow equations, computer simulations were run to study shear stress magnitude and distribution over time. The average inlet velocity used at the entrance of a single scaffold pore was $(0.0012 \mathrm{~m} / \mathrm{s})$ and corresponded to a $1 \mathrm{ml} / \mathrm{min}$ perfusion flow rate for the geometric features of the bioreactor. Figure 3 shows the gradual closure and the development of the correspondingly changing flow profiles for the two different scaffold designs. Shear stress values were determined both at the interface of the neotissue with the free flow domain and also within the neotissue itself. For the square design, the average interface shear stress values evolved from 0.0034 to 0.005 Pa while for the diamond design the average interface shear stress values evolved from 0.004 to $0.008 \mathrm{~Pa}$ during the filling of the scaffold macro-pores. Interface shear stress values reported here ranged from being comparable (for i.e. 10\% filling) to two orders of magnitude higher (for i.e. $60 \%$ filling upwards) compared to the ones calculated for empty scaffold geometries in CFD modeling studies to date, as seen in Table 1. The average shear stresses developed within the growing neotissue were higher as expected, evolving from 0.04 to 0.28 $\mathrm{Pa}$ in the diamond pore shape scaffold and from 0.03 to $0.18 \mathrm{~Pa}$ in the square pore shape scaffold during the filling of the scaffold macro-pores. Additionally, the absolute values of the shear stresses were dependent on the micro-porosity of the neotissue domain (Figure 4). 
Figure 5 shows the shear stress distribution within the neotissue for different degrees of scaffold filling. Following this distribution for different degrees of scaffold filling (different colors), it demonstrates the shift from lower towards higher shear stress values within the neotissue due to the scaffold filling. The geometric homogeneity of the square pore scaffold was reflected on the shear stress distribution inside the neotissue which was narrower for the square pore scaffolds than for the diamond pore scaffolds. Furthermore it should be noted that average inner shear stress values calculated here, especially in conditions with high degrees of scaffold filling, were closer to calculated values experienced by cells in the in vivo setting, reported to range from $0.8 \mathrm{~Pa}$ onwards (Weinbaum, Cowin et al. 1994) or, as calculated more recently, from 0.1-10 Pa experienced by osteocytes due to fluid flow via canaliculi (Verbruggen, Vaughan et al. 2014). Interestingly, these shear stress levels also closely compared with recent work focusing on shear stresses experienced by single cells bridging across scaffold pore struts (Jungreuthmayer, Jaasma et al. 2009; Zhao, Vaughan et al. 2014).

In this study a number of simplifications were made that warrant further investigation in future work. First and foremost it will be necessary to couple the shear stress experienced by the cells (and calculated in this study) to the neotissue growth rate. Various studies have shown the influence of mechanical stimuli on the behavior of cells (McCoy and O'Brien 2010; Rauh, Milan et al. 2011; Papantoniou, Chai et al. 2013 to but name a few of them). Adding a more detailed description of the neotissue constituents (cells and extracellular matrix (ECM)) and/or additional variables such as oxygen and glucose will allow to capture additional biological behavior observed in bioreactor experiments. These observations include the decrease in neotissue micro-porosity due to ECM production, the cellular lineage commitment and the oxygen consumption during culture. 


\section{Conclusion}

In conclusion, this framework provides insight into the dynamic microenvironment to which cells are exposed in TE perfusion bioreactors. The development of such a model generates an additional level of control by allowing operators to deliver controlled shear stress magnitudes to cells during growth, e.g. by adapting flow rates during neotissue growth in order to maintain fixed shear stress magnitudes and profiles causing minimal culture environment variation. Moreover this model could be used for an in silico evaluation and screening of scaffold designs, including their impact on neotissue growth and exposure to stress profiles, avoiding costly and time consuming in vitro experiments.

\section{Acknowledgments}

YG is funded by Belgian National Fund for Scientific Research (FNRS) grant FRFC 2.4564.12. IP is funded by the European Research Council under the European Union's Seventh Framework Programme (FP/2007-2013) / ERC Grant Agreement n. 294191. The research leading to these results has received funding from the European Research Council under the European Union's Seventh Framework Programme (FP/2007-2013) / ERC Grant Agreement n. 279100 and the FWO-ENDEAVOUR project. This work is part of Prometheus, the Leuven Research \& Development Division of Skeletal Tissue Engineering of the KU Leuven: www.kuleuven.be/prometheus. 


\section{References}

Bidan, C. M., F. M. Wang, et al. (2013). "A three-dimensional model for tissue deposition on complex surfaces." Comput Methods Biomech Biomed Engin.

Boschetti, F., M. T. Raimondi, et al. (2006). "Prediction of the micro-fluid dynamic environment imposed to three-dimensional engineered cell systems in bioreactors." Journal of Biomechanics 39(3): 418-425.

Cioffi, M., F. Boschetti, et al. (2006). "Modeling evaluation of the fluid-dynamic microenvironment in tissue-engineered constructs: A micro-CT based model." Biotechnology and Bioengineering 93(3): 500-510.

Eyckmans, J. and F. P. Luyten (2006). "Species specificity of ectopic bone formation using periosteum-derived mesenchymal progenitor cells." Tissue Eng 12(8): 2203-2213.

Guyot, Y., I. Papantoniou, et al. (2014). "A computational model for cell/ECM growth on 3D surfaces using the level set method: a bone tissue engineering case study." Biomech Model Mechanobiol.

Hecht F (2012) New development in freefem++. $J$ Numer Math 20(4):251-265. doi:10.1515/jnum-2012-0013

Hendrikson, W. J., C. A. van Blitterswijk, et al. (2014). "Modeling mechanical signals on the surface of microCT and CAD based rapid prototype scaffold models to predict (early stage) tissue development." Biotechnol Bioeng 111(9): 1864-1875.

Hutmacher, D. W. and H. Singh (2008). "Computational fluid dynamics for improved bioreactor design and 3D culture." Trends Biotechnol 26(4): 166-172.

Joly, P., G. N. Duda, et al. (2013). "Geometry-driven cell organization determines tissue growths in scaffold pores: consequences for fibronectin organization." PLoS One 8(9): e73545.

Jungreuthmayer, C., S. W. Donahue, et al. (2009). "A Comparative Study of Shear Stresses in Collagen-Glycosaminoglycan and Calcium Phosphate Scaffolds in Bone TissueEngineering Bioreactors." Tissue Engineering Part A 15(5): 1141-1149.

Jungreuthmayer, C., M. J. Jaasma, et al. (2009). "Deformation simulation of cells seeded on a collagen-GAG scaffold in a flow perfusion bioreactor using a sequential 3D CFDelastostatics model." Med Eng Phys 31(4): 420-427.

Lesman, A., Y. Blinder, et al. (2010). "Modeling of flow-induced shear stress applied on 3D cellular scaffolds: Implications for vascular tissue engineering." Biotechnol Bioeng 105(3): 645-654.

Maes, F., P. Ransbeeck, et al. (2009). "Modeling Fluid Flow Through Irregular Scaffolds for Perfusion Bioreactors." Biotechnology and Bioengineering 103(3): 621-630.

Martin, I., D. Wendt, et al. (2004). "The role of bioreactors in tissue engineering." Trends Biotechnol 22(2): 80-86. 
McCoy, R. J. and F. J. O'Brien (2010). "Influence of Shear Stress in Perfusion Bioreactor Cultures for the Development of Three-Dimensional Bone Tissue Constructs: A Review." Tissue Engineering Part B-Reviews 16(6): 587-601.

Nabovati, A., Llewellin, E.W, Sousa, A.C.M. (2009). "A general model for the permeability of fibrous porous media based on fluid flow simulations using the lattice Boltzmann method." Composites: Part A 40: 860-869.

Nava, M. M., M. T. Raimondi, et al. (2013). "A multiphysics 3D model of tissue growth under interstitial perfusion in a tissue-engineering bioreactor." Biomech Model Mechanobiol 12(6): 1169-1179.

Papantoniou, I., Y. C. Chai, et al. (2013). "Process Quality Engineering for Bioreactor-Driven Manufacturing of Tissue-Engineered Constructs for Bone Regeneration." Tissue Engineering Part C-Methods 19(8): 596-609.

Papantoniou, I., Y. Guyot, et al. (2014). "Spatial optimization in perfusion bioreactors improves bone tissue-engineered construct quality attributes." Biotechnol Bioeng.

Papantoniou, I., M. Sonnaert, et al. (2014). "Three-dimensional characterization of tissueengineered constructs by contrast-enhanced nanofocus computed tomography." Tissue Eng Part C Methods 20(3): 177-187.

Papantoniou, I. I., Y. C. Chai, et al. (2013). "Process Quality Engineering for Bioreactor-Driven Manufacturing of Tissue-Engineered Constructs for Bone Regeneration." Tissue Eng Part C Methods.

Pham, N. H., R. S. Voronov, et al. (2012). "Predicting the stress distribution within scaffolds with ordered architecture." Biorheology 49(4): 235-247.

Porter, B., R. Zauel, et al. (2005). "3-D computational modeling of media flow through scaffolds in a perfusion bioreactor." Journal of Biomechanics 38(3): 543-549.

Pyka, G., A. Burakowski, et al. (2012). "Surface Modification of Ti6Al4V Open Porous Structures Produced by Additive Manufacturing." Advanced Engineering Materials 14(6): 363-370.

Raimondi, M. T., F. Boschetti, et al. (2004). "The effect of media perfusion on three-dimensional cultures of human chondrocytes: Integration of experimental and computational approaches." Biorheology 41(3-4): 401-410.

Rauh, J., F. Milan, et al. (2011). "Bioreactor systems for bone tissue engineering." Tissue Eng Part B Rev 17(4): 263-280.

Song, M. J., D. Dean, et al. (2013). "Mechanical modulation of nascent stem cell lineage commitment in tissue engineering scaffolds." Biomaterials 34(23): 5766-5775.

Sonnaert, M., I. Papantoniou, et al. (2014). "Human periosteal-derived cell expansion in a perfusion bioreactor system: proliferation, differentiation and extracellular matrix formation." J Tissue Eng Regen Med.

Van Bael S, Chai YC, Truscello S, Moesen M, Kerckhofs G, Van Oosterwyck H, Kruth JP, Schrooten J (2012) The effect of pore geometry on the in vitro biological behavior of 
human periosteum-derived cells seeded on selective laser-melted Ti6Al4V bone scaffolds. Acta Bio mater 8(7):2824-2834. doi:10.1016/j.actbio.2012.04.001

Verbruggen, S. W., T. J. Vaughan, et al. (2014). "Fluid flow in the osteocyte mechanical environment: a fluid-structure interaction approach." Biomech Model Mechanobiol 13(1): 85-97.

Voronov, R., S. VanGordon, et al. (2010). "Computational modeling of flow-induced shear stresses within 3D salt-leached porous scaffolds imaged via micro-CT." Journal of Biomechanics 43(7): 1279-1286.

Voronov, R. S., S. B. VanGordon, et al. (2013). "3D Tissue-Engineered Construct Analysis via Conventional High-Resolution Microcomputed Tomography Without X-Ray Contrast." Tissue Engineering Part C-Methods 19(5): 327-335.

Weinbaum, S., S. C. Cowin, et al. (1994). "A model for the excitation of osteocytes by mechanical loading-induced bone fluid shear stresses." Journal of Biomechanics 27(3): 339-360.

Whittaker, R. J., R. Booth, et al. (2009). "Mathematical modelling of fibre-enhanced perfusion inside a tissue-engineering bioreactor." J Theor Biol 256(4): 533-546.

Zhao, F., T. J. Vaughan, et al. (2014). "Multiscale fluid-structure interaction modelling to determine the mechanical stimulation of bone cells in a tissue engineered scaffold." Biomech Model Mechanobiol. 


\begin{tabular}{|c|c|c|c|c|}
\hline & & $\begin{array}{l}\text { Scaffold Pore } \\
\text { size }(\mu \mathrm{m})\end{array}$ & $\begin{array}{c}\text { Average Shear } \\
\text { Stress (mPa) }\end{array}$ & $\begin{array}{c}\text { Inlet velocity } \\
(\mu \mathrm{m} / \mathrm{s})\end{array}$ \\
\hline \multirow{7}{*}{$\begin{array}{l}\text { Wall } \\
\text { shear } \\
\text { stress }\end{array}$} & $\begin{array}{c}\text { (Maes, Ransbeeck } \\
\text { et al. 2009) }\end{array}$ & 280 & 1.4 & 34 \\
\hline & $\begin{array}{c}\text { (Cioffi, Boschetti } \\
\text { et al. 2006) }\end{array}$ & 100 & $3.28-3.94$ & 53 \\
\hline & $\begin{array}{c}\text { (Lesman, Blinder } \\
\text { et al. 2010) }\end{array}$ & 500 & 11 & 5000 \\
\hline & $\begin{array}{l}\text { Nava (Nava, } \\
\text { Raimondi et al. } \\
\text { 2013) }\end{array}$ & 300 & 5 & 100 \\
\hline & $\begin{array}{l}\text { (Zermatten, } \\
\text { Vetsch et al. } \\
\text { 2014) }\end{array}$ & 220 & 3.08 & 66 \\
\hline & $\begin{array}{l}\text { (Shakhawath } \\
\text { Hossain 2015) }\end{array}$ & 1000 & 40 & 230 \\
\hline & $\begin{array}{c}\text { This study } \\
\text { (interface shear } \\
\text { stress) }\end{array}$ & 600 & $3.4-8$ & 1200 \\
\hline Signle & $\begin{array}{c}\text { (Zhao, Vaughan } \\
\text { et al. 2014) }\end{array}$ & 180 & $50-250$ & 300 \\
\hline
\end{tabular}




\begin{tabular}{|c|c|c|c|c|}
\hline $\begin{array}{c}\text { cells / } \\
\mu- \\
\text { porosity / }\end{array}$ & $\begin{array}{l}\text { (Jungreuthmayer, } \\
\text { Jaasma et al. } \\
\text { 2009) }\end{array}$ & 96 & 30 & 235 \\
\hline $\begin{array}{l}\text { inner } \\
\text { shear } \\
\text { stress }\end{array}$ & This study & 600 & $10-250$ & 1200 \\
\hline
\end{tabular}

Table 1:A comparison between empty scaffold shear stress values calculated in literature and interface shear stress values obtained in this study. In the second group the inner shear stresses obtained for the neotissue part were compared to literature shear stress values for bridging single cell models,please note that shear stress units are in $\mathrm{mPa}$.All shear stress presented have been obtained for flow-through perfusion bioreactors. 
Figure 1: (A) Image indicating the the perfusion rig dimensions $\left(D_{\text {rig }}=6 \mathrm{~mm}, L_{\text {rig }}=26 \mathrm{~mm}\right)$ and scaffold position which was $\mathrm{L}_{\mathrm{entr}}=10 \mathrm{~mm}$ from the entrance of the rig. Both scaffolds had the same external dimensions $D_{\text {scaf }}=6 \mathrm{~mm}$ and $\mathrm{L}_{\text {scaf }}=6 \mathrm{~mm}$, also the same strut thickness was used for both pore designs were $\mathrm{D}_{\text {strut }}=200 \mu \mathrm{m}$. (B) Diamond scaffold pore size was $750 \mu \mathrm{m}$ from the center of one strut to the other as shown by the dashed arrows. (C) Square pore scaffold pore size was $650 \mu \mathrm{m}$.

Figure 2: Live/dead staining indicating three dimensional neotissue growth during culture over a culture period of 21 days in perfusion bioreactors in two different scaffolds designs: (A) diamond and (b) square. In both cases neotissue eventually fills the scaffold void by day 21, (scale bar $=800 \mu \mathrm{m}$ ). SEM shows the micro-porosity of the growing neotissue (representative image).

Figure 3: $(\mathrm{A}, \mathrm{C})$ Inner and $(\mathrm{B}, \mathrm{D})$ interface shear stress magnitude and distribution during neotissue growth and gradual filling of the void in scaffolds with $(A, B)$ square and $(C, D)$ diamond pore shape. Neotissue micro-porosity used for this simulation was $50 \mu \mathrm{m}$.

Figure 4: Average inner (blue bars) and interface (red bars) shear stress values for both scaffold geometries and neotissue micro-porosities investigated in this study for a range of neotissue scaffold void filling percentages.

Figure 5: Shear stress distribution within the neotissue during growth. Different colors represent the filling percentage of the scaffold void during neotissue growth, ranging from $10 \%$ to $90 \%$ as indicated in the figure. 




Figure 1 




Figure 2

This article is protected by copyright. All rights reserved 


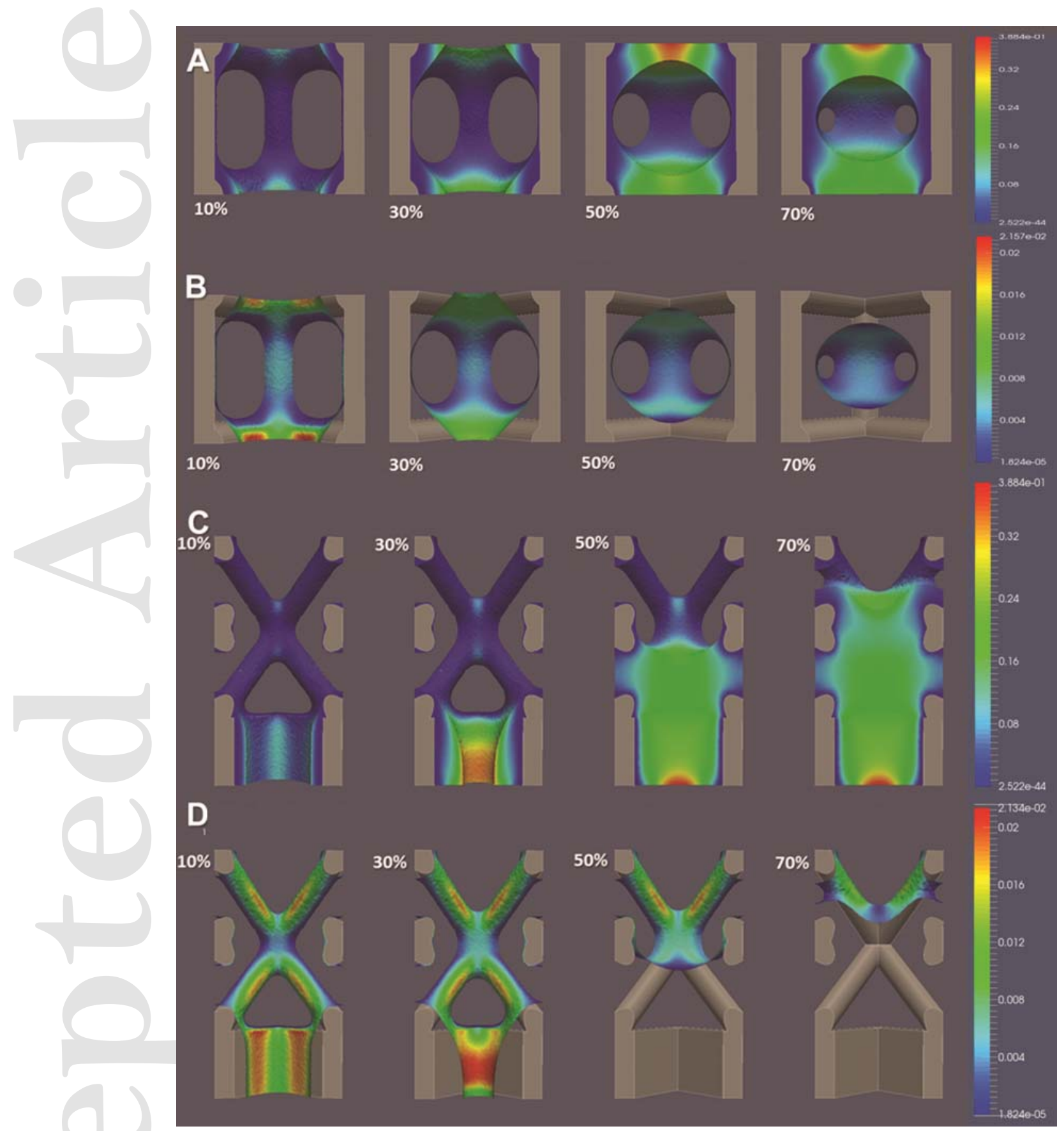

Figure 3 


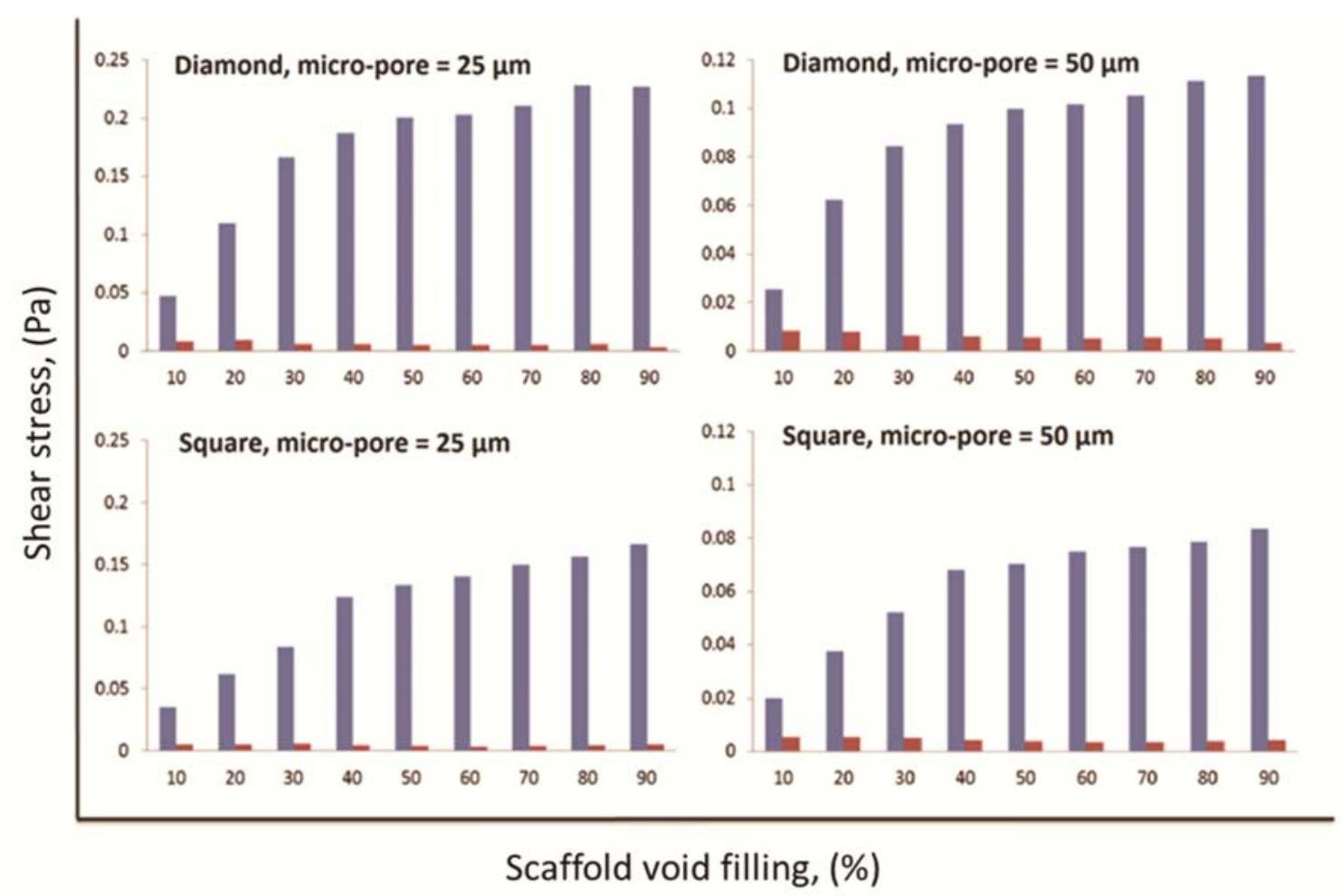

Figure 4 


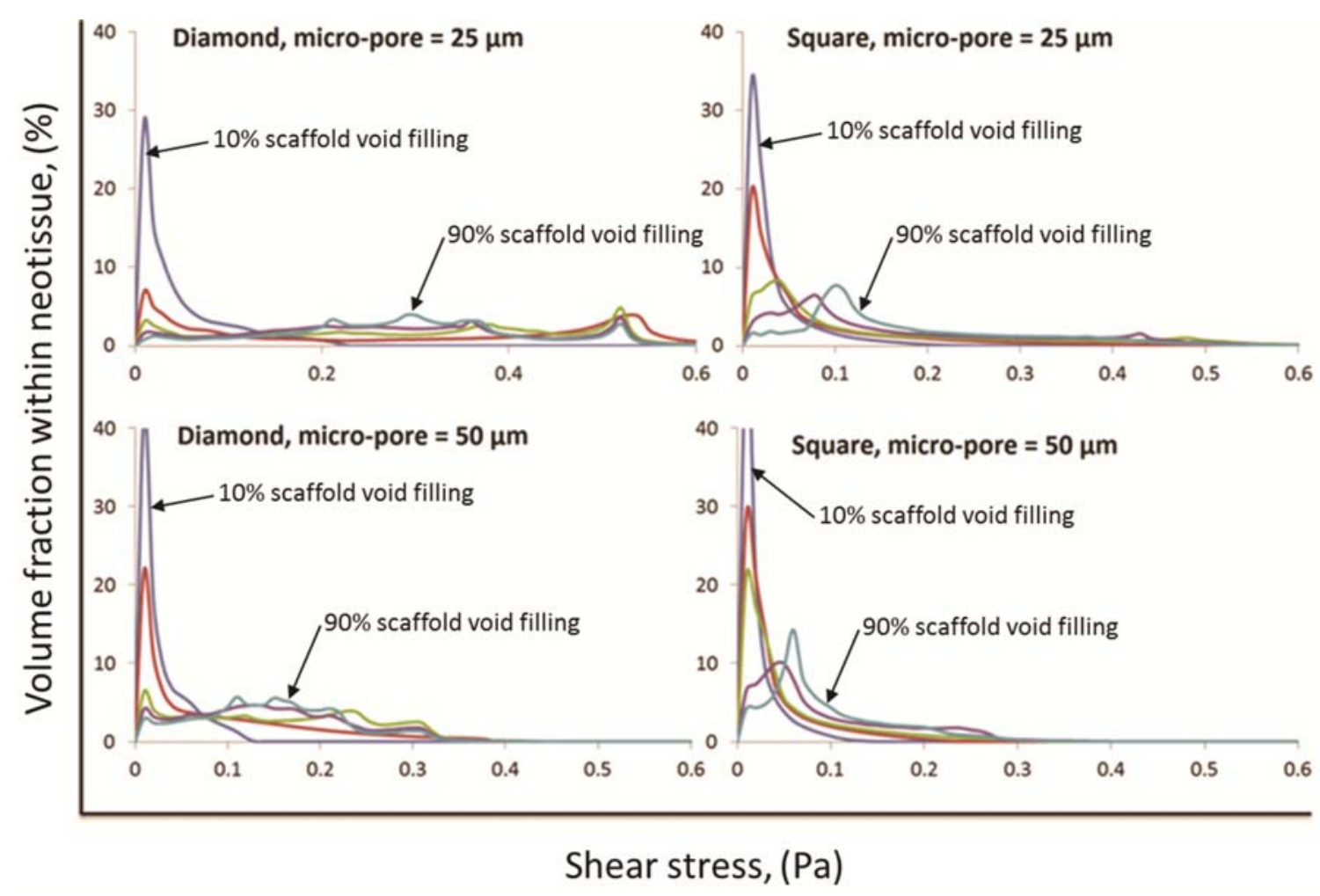

Figure 5

This article is protected by copyright. All rights reserved 